\title{
Hypoxia Inducible Factor Family
}

National Cancer Institute

\section{Source}

National Cancer Institute. Hypoxia Inducible Factor Family. NCI Thesaurus. Code

C113839.

A family of basic helix-loop-helix heterodimeric transcription factors that activate gene expression when oxygen levels decrease in the cellular environment. Each HIF complex is comprised of an alpha subunit and beta subunit, which are encoded by separate genes. 